\title{
Review
}

Psychopathology

Psychopathology 2012;45:337-343

DOI: $\underline{10.1159 / 000337748}$
Received: June 14, 2011

Accepted after revision: February 25, 2012

Published online: July 31, 2012

\section{Reduplicative Paramnesia: A Review}

\author{
Marios Politis Clare Loane \\ Centre for Neuroscience, Division of Experimental Medicine, Faculty of Medicine. Hammersmith Hospital, \\ Imperial College London, London, UK
}

\section{Key Words}

Reduplicative paramnesia - Delusional misidentification

syndromes · Neurology

\begin{abstract}
Background: Reduplicative paramnesia (RP) is a contentspecific delusional misidentification syndrome (DMS) which has received little attention in the research literature relative to other DMS. RP is thought to result from an organic rather than psychiatric cause distinguishing it from other DMS. Our systematic review examines the research literature investigating the prevalence, symptomatology and potential neurologic mechanisms underlying RP. Sampling and Methods: MEDLINE, PsycINFO, and the Cochrane Library were searched (from 1966 to February 10, 2012) with the reference lists of relevant articles examined. Case reports, clinical studies and post-mortem studies focusing on, or referring to, RP were included. Results: There is a paucity of literature regarding the potential mechanisms underlying the psychological, cognitive and neurological aspects of RP. The available literature is limited by the lack of systematic clinical studies and in vivo investigations with current findings remaining only speculative. However, there does appear to be a consensus that RP may have a neurologic rather than psychiatric cause and that right and bifrontal lesions as well as the cognitive dissonance associated with memory, visuospatial and impaired conceptual integration are common factors in RP
\end{abstract}

presentation. Conclusions: This area requires further extensive systematic research with supplementary in vivo data. Current studies suggest that focal lesions within the frontal lobe may account for the onset of RP.

Copyright $\odot 2012$ S. Karger AG, Basel

\section{Introduction}

A subset of the delusional misidentification syndromes (DMS) is characterised by the subjective conviction of an individual, that a place, person or event is duplicated and as such, these are described as reduplicative misidentification syndromes (RMS). The subjective belief that a place has been duplicated, existing in at least two locations simultaneously, is termed reduplicative paramnesia (RP) and unlike other duplicative syndromes, is thought to be mainly due to a neurologic cause. As such, RP will be the main focus of this review. The term RP was first used by the neurologist Arnold Pick in 1903 whilst describing his observation of the phenomenon in a female patient with suspected neurodegenerative disease [1]. Pick's patient claimed that the Prague clinic she was being treated in was in fact an exact replica of the clinic in her home town and that she was actually in the 'duplicate' clinic in her home town and not Prague. When questioned about the irregularities of such a possibility she resisted all attempts to dissuade her that her belief was

\section{KARGER}

Fax +4161306 1234

E-Mail karger@karger.ch

www.karger.com
(C) 2012 S. Karger AG, Basel

0254-4962/12/0456-0337\$38.00/0

Accessible online at:

www.karger.com/psp
Marios Politis

Hammersmith Hospital

London W12 0NN (UK)

Tel. +44208383 3751

E-Mail marios.politis@imperial.ac.uk 
incorrect and insisted that Pick and the other hospital staff worked at both locations to explain the discrepancies. It is a common characteristic of RP patients to remain certain that their claims are correct, even when plausible explanations of their belief are presented to them. However, looking retrospectively it appears that the phenomenon was identified much earlier by the naturalist Charles Bonnet in 1788 [2]. Bonnet described an elderly female who believed that she was dead (a phenomenon now termed, Cotard's delusion) [3] in combination with the steadfast belief that her current location was in another place (RP). Later cases of RP were reported regarding soldiers that had suffered a traumatic brain injury during duty and held the belief that the hospital they were being treated in was located in their hometown [4, 5]. From these cases, the brain injury was attributed to the development of RP. Unlike other DMS, the pathogenesis of RP appears to have a significant neurologic component, rather than psychological. However, due to technical limitations, investigations of neurological causes of RP in vivo have not been possible until relatively recently. Serious consideration of the neurological as well as psychological mechanisms of RP was initiated in 1976 by Benson and colleagues [6]. Following this revival of interest in RP, there have been several attempts to delineate the underlying causes of RP via case studies as well as a few small research studies. This review will describe what is known about RP to date from its symptomatology to pathogenesis to treatment.

\section{Definition and Epidemiology}

RP shares features with other DMS (i.e. Capgras and Fregoli syndromes) in that it is characterized by the idea of 'doubles' and is a content-specific delusion. The core feature of RP is typified by the subjective belief that a place has been duplicated and exists simultaneously in two or more locations. There are three variants of RP which may present alone or coexist. 'Place reduplication' refers to the belief that two places with identical features exist simultaneously, but are geographically distant, which is the variant identified in Pick's early case study. 'Chimeric assimilation' presents as two places becoming combined, for example, a patient in hospital believes that they are in their own home which has somehow transformed into the hospital. Finally 'extravagant spatial localisation' presents as a patient believing that their current location is actually somewhere else, usually a location familiar to them.
Another condition similar to RP, which also is related to the subjective feeling of duplication is that of 'invisible Doppelgänger' which can be described as an individual believing they have a clone or indeed believing that they themselves are a clone of their 'real' self. Doppelgänger is a concept which has received attention in popular culture, fiction and folklore and is a term which has become diluted in many languages to simply describe a double or look-alike of an individual. However, it is suggested to also be of a neurologic cause, similar to RP and so will be covered in this review.

There is currently no epidemiology data regarding RP. This reflects the general paucity of epidemiological estimate of all DMS with tentative figures reported based on retrospective reviews and single case studies. However, content-specific delusions (of which RP is one) have been suggested as occurring in approximately $>1-5.3 \%$ of people within the psychiatric setting $[7,8]$. Of course, these estimates encompass all the variants of DMS which are likely to have varying underlying mechanisms ranging from psychiatric to neurological and therefore, must be viewed cautiously. Furthermore, there is no attempt to delineate the proportion of RP cases included in this estimate. To date, the only attempt to estimate the prevalence of RP was born out of a small sample of inpatient alcoholics demonstrating the RP phenomenon, where it was reported that an estimated $8 \%$ of this population present with RP [9]. Systematic assessment and reporting of the RP phenomenon is required to establish accurate epidemiological data.

\section{Signs and Symptoms}

Patients with RP are able to recognise familiar places or landmarks adequately, but appear to have difficulty orienting the location of these places in relation to the self accurately. As previously described, this difficulty may manifest in at least one of three beliefs; place reduplication, chimeric assimilation and extravagant spatial localisation. Aside from the delusional belief of duplication, a spectrum of cognitive and behavioural disturbances can be observed in RP patients. It is thought that there are three clusters of cognitive deficits necessary for RP, specifically, memory deficits, impaired geographical/visuospatial skills and executive skill dysfunction [10]. RP patients often appear to show little concern regarding their condition and decreased foresight [11]. Confabulation is also common and usually resolves relatively quickly after the initial injury. 


\section{Diagnosis and Differential Diagnosis}

$\mathrm{RP}$ is reported to occur following a variety of neurological conditions including head trauma and cerebral infarction [11], toxic and posttraumatic encephalopathies, tumours in the third ventricle and aneurysm ruptures involving the circle of Willis [9]. It is interesting to note that unlike most DMS, RP is usually attributed to an organic cause rather than being of psychiatric or psychological origin. Improved diagnostic techniques have also produced an increase in the accuracy in identifying neurologic disease in those presenting with delusions [12] including RP.

However, the diagnosis of RP may not be straightforward due to similarities between various other delusions and perceptual disturbances commonly observed in various neurological conditions. For example, following a brain trauma it is common for patients to experience confusion, confabulate and suffer memory disturbances, particularly when the frontal lobe is involved. It is important when diagnosing RP to distinguish what type of delusion is taking place as it is common for patients to experience a period of 'misperception' following brain trauma. There are features of RP delusions which enable differentiation from other disturbances of cognition that may not actually be a delusional belief. For example, the delusion must be persistent and resistant to any attempts to persuade the patient their beliefs are inaccurate or impossible despite clear evidence on the contrary [11]. Other visual agnosias such prosopagnosia and simultanagnosia, the inability to recognise faces and objects, respectively, are less likely to be considered delusional beliefs as these patients do not insist that the person or object in question is an imposter and are existing elsewhere but simply have a failure of recognition. Furthermore, prosopagnosia may be considered the opposite to Capgras syndrome, another condition which is characterised by the concept of 'doubles' similar to RP. Recent neurophysiological evidence has suggested that prosopagnosia results following a disconnection of the links between intact identity-specific visual memory traces and later semantic face processing stages as well as suggesting that memory storage of recognized faces is insufficient in cases of prosopagnosia [13]. In addition, simultanagnosia has also been attributed to the disruption of the visuospatial attention network [14].

Distinguishing 'delusional' features between these various disorders are intricate and often present simultaneously in clinical practice. It is important to monitor the changes of such behaviours as perceptual disorders commonly present initially but turning into persistent delusional beliefs. Another important distinction to make is between content-specific delusions and primary psychiatric disorders such as paranoid schizophrenia. The most obvious difference is that those with paranoid delusions will behave suspiciously and be strongly defensive of their beliefs when challenged, whereas patients with RP (or any other content-specific delusion) are usually described as approachable and cooperative and will admit bewilderment regarding the implausibility of their beliefs despite maintaining that they are true. As such, it appears to be vital that the RP phenomena presenting in the context of other psychosis symptoms, such as paranoia, are considered as part of a psychiatric illness and not attributed to an organic cause. Therefore, RP symptomatology presenting alone is likely to be attributed to an organic cause.

Confabulation and anosognosia are closely related to delusions, but differ in time course, presentation and the neuroanatomy involved. Confabulation is a memory disturbance frequently observed in patients following brain trauma and often coexists with RP and other content-specific delusions. Patients who confabulate will present false beliefs and memories but lack any intent to deceive. However, these patients tend to be relatively easy to correct and when the two symptoms coexist the confabulatory behaviour typically resolves first $[15,16]$. Confabulation and RP are associated with different neuroanatomical regions. It has been reported that confabulation is associated with ventromedial and orbitofrontal lesions $[6,17]$ with the confabulatory behavior occurring and resolving during the same time course as the lesions. On the other hand, the occurrence of RP appears to be consistent with right hemispheric and/or bifrontal pathology. Anosognosia is a condition in which an individual who suffers a disability appears to be unaware that the disability exists. It is a relatively common condition following brain injury and it is not simply the manifestation of denial as a defense mechanism but has its roots in physiology. It is thought that anosognosia occurs due to disruption of higher neurocognitive processes which integrate sensory information with spatial or bodily representations [18]. The idea of a loss of 'sensory rationality' is similar to the concept of RP. Furthermore, it is a condition which is usually connected to the nondominant hemisphere (typically the right hemisphere), again similar to RP.

Improving clarity regarding the differential diagnosis of RP is pertinent in the clinic to ensure the correct diagnosis is made in the first instance. However, for an effective and precise treatment regime to be identified, further research is required. Possible insights may be gained from similar disorders of perception such as those outlined above. Impairment of visuospatial skills, memory and ex- 
Table 1. Studies of RP and anatomical and neuropsychological findings

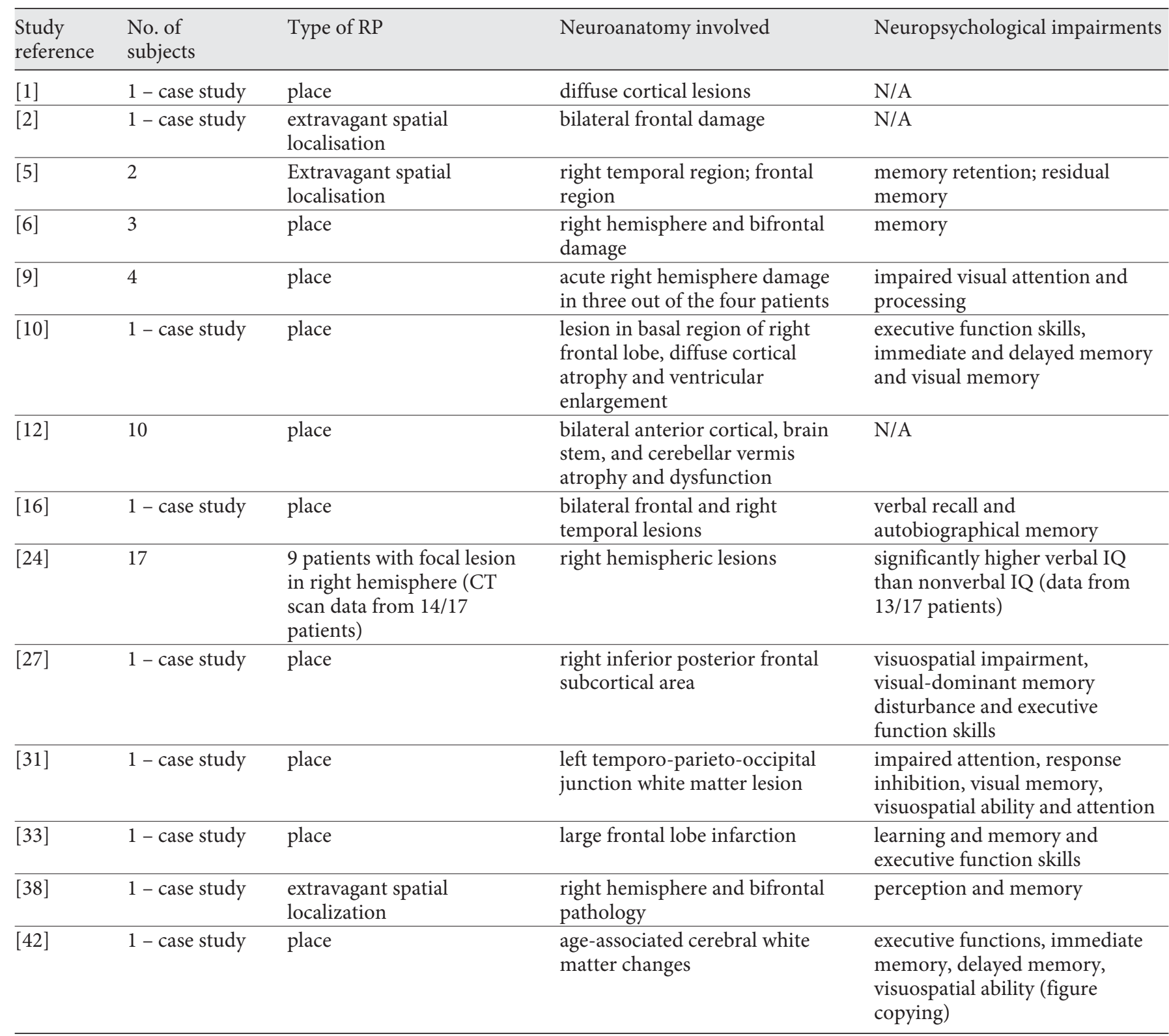

N/A = Data not available; CT = computerised tomography; IQ = intelligence quotient.

ecutive function skills all appear to be involved in the various agnosias although each resulting in a different condition.

Finally, Doppelgänger has been likened to the 'phantom limb' phenomenon, whereby an amputee distinctly recounts descriptions of their missing limb occupying its usual space and is considered an integral part of one's self [19]. The vividness, three-dimensionality and affinity to the self are common characteristics between the two con- cepts and as such have been considered to share a neurologic cause [20]. Doppelgänger has been attributed to arise via a disturbance in a (currently, relatively unknown) neuroanatomical substratum responsible for the self-experience of owning a body [19]. It is supposed that this process is located diffusely across the brain and particularly related to thalamocortical and limbic loops. Lateralisation, similar to RP, appears to be important in the presentation of Doppelgänger experience. For example, 
when reduplicative symptoms present to the right side of the patient, i.e. the Doppelgänger appears on the right side, insight appears to be preserved. However, when symptoms present on the left side, this insight appears to be lost [unpublished data mentioned in 21].

Neuroimaging studies and in-depth neuropsychological assessment may reveal information not only for the condition under investigation but also its related conditions including RP.

\section{Pathophysiology and Pathology}

Authors have described RP after lesioning in the right hemisphere and/or bifrontal areas [11, 22-27]. For example, Benson et al. [25] suggested that the right hemispheric damage they report resulted in an impairment of visuospatial perception and visual memory, whereas the damage reported in the frontal lobe made it difficult to inhibit the false impressions caused by disorientation. Variable parietal and temporal contribution has also been reported $[6,26,28]$.

Studies assessing the neuropathological correlates of RP are limited with most early descriptions arising from single case studies. Most recently, RP has been investigated with diffusion-weighted imaging (DWI) in a 69 -year-old male with a localised lesion in the right inferior posterior frontal subcortical area [29]. Using DWI, the authors were able to deduce five neural pathways which may have been disrupted due to lesion citing four of which may be involved in the etiology of RP in this case: the superior longitudinal fasciculus, fronto-occiptal fasciculus (both related to visuospatial processing), anterior thalamic radiation (memory processes) and uncinate fasciculus (sensations of hyperfamiliarity). Forstl et al. [24] have produced a report assessing the largest number of RP in one study by reviewing 260 cases of DMS which included 17 cases of RP. Although the individual details of these cases were not presented in this review, it was stated that the cases of RP displayed more evidence of right hemisphere lesions (determined through computerised tomography (CT) scans and neurophysiological testing) compared to the cases presenting with other DMS. A recent review [30] reported the neuropathology of 69 cases of RP, 36 of which (52\%) had a primary lesion in the right hemisphere, in 28 cases (41\%) bi-lateral pathology was found and in just 5 cases (7\%) a left hemisphere lesion was identified. This seven-fold increase of right over left lesions ( $\mathrm{p}<0.0001)$ supports previous findings. A similar pattern was found in the same review in cases of Capgras syndrome, although the right over left prevalence was smaller at a fourfold increase $(p<0.006)$. However, for both syndromes, cases with bilateral pathology had the greatest damage in the right hemisphere. However, this does not explain how lesions to the left hemisphere alone may result in RP. Budson et al. [31] reported a case study of a left-handed 45-year-old man with a history of alcoholism who was admitted to hospital for a minor operation and suffered a small left hemisphere stroke. During his admittance, he was reported to have become confused regarding his orientation alone, i.e. he did not display any other forms of confusion. The patient insisted that he had another room in the hospital which was located in the hallway. Typically of RP, the patient could not explain why he would have another room in the hallway but maintained his insistence that it existed. The stroke damaged white matter adjacent to Brodmann areas 19 and 37 likely disrupting the occipitotemporal projection system which is the fibre pathway of the ventral visual stream. Neuropsychological testing revealed impairments in visuospatial perception and memory, which are usually associated with the nondomainant hemisphere, tying in with the fact that this patient was left-handed.

RP may develop preferentially following right hemisphere lesion due to two possibilities: (a) loss of self-related function monitoring of reality, familiarity and memory $[26,28,32]$ or (b) overactivity in the left hemisphere due to a right hemisphere deficit. The right hemisphere dominates awareness and image of self and it is the integration of relating the self to external and internal environments $[26,28,32]$. Therefore, it is proposed that right frontal lesions can disconnect temporolimbic structures, leading to the preservation of familiarity of places but an inability to accurately identify its location to the self [33]. An interruption between fronto-parieto-temporal areas and the limbic areas may dissociate the place with its emotional significance. Although there is no anatomical or theoretical evidence suggesting that this circuit may be lateralized to the right hemisphere, the evidence of RP occurring after right hemisphere lesions indicates that at least some processes of recognition lie within the right hemisphere.

It has been suggested that the temporal lobe may be responsible for determining the type of delusion experienced. It has been reported in a review of DMS (not specifically RP) that temporal lesions were found in $64 \%$ of patients who experienced delusions whereby a place, person or object was unfamiliar compared to just $14 \%$ of delusions relating to the misidentification of people, places and objects as familiar. This suggests that temporal lesioning (and not sparing) may be more likely to pro- 
duce a case of RP [for review, see 30]. It has been proposed that the right posterior temporoparietal region may be responsible for the recognition and familiarity of a place, and therefore, a lesion in this region may cause a disruption in this recognition process resulting in $\mathrm{RP}$ [6]. Another region that is known to be responsible for the recognition of scenes is the parahippocampal cortex, which may be involved in the underlying mechanism of RP. The anterior parahippocampal cortex (perirhinal cortex, Brodmann areas 35 and 36) is activated by familiarity. The hippocampus and posterior parahippocampal cortex are responsible for mediating familiarity [34]. It is plausible that a lesion resulting in the isolation of stimuli from the perirhinal cortex in the right hemisphere could possibly lead to a loss in familiarity.

Pick (the originator of the RP term) attributed the pathogenesis of his RP patient to be diffusely cortical. This finding has been supported $[22,35,36]$ with one author proposing diffuse lesions may cause a disruption of the interconnections between the frontal cortex, thalamus and reticular formation [36]. Furthermore, RP has been described in cases whereby there is widespread brain lesioning rather than a focal infarct without generalized atrophy and/or bifrontal involvement [22]. It has been proposed that a frontal lesion may contribute to the development of RP, but for the cognitive-perceptual-affective dissonance of RP to occur, wider neurological dysfunction is required [35].

The underlying pathology of Doppelgänger has also been investigated with a recent diffusion tensor imaging (DTI) study revealing in an otherwise healthy 48-yearold man the development of Doppelgänger following a small lesion in the fronto-opercular region [29]. The authors suggest that the Doppelgänger presentation was due to a far-reaching 'diaschisis'-like effect of the frontoopercular acute lesion on parietal functions via the ventral bundle of the superior longitudinal fasciculus, and although the lesion was small, it was sufficient enough to cause the temporary sensation of cloning of the self.

\section{Treatment and Management}

$\mathrm{RP}$ often resolves spontaneously usually after a period of rehabilitation and reorientation [6, 37]. However, there is paucity in the literature on the effective management or treatment of RP. A few case reports have cited resolution of the syndrome following low doses of haloperidol [38] and also fluphenazine hydrochloride when haloperidol was ineffective [39]. Controlled clinical trials need to be performed to identify effective treatments for these delusions. A related phenomenon, déjà vu (the illusion of recognition and subjective impression of familiarity of the present with an undefined point in the past) has been suggested to be associated with an increase in dopamine (DA) activity [40]. In this case study, the authors present a patient who was treated for influenza with a combination therapy of amantadine and phenylpropanolamine. Both of these agents facilitate increased DA turnover. During treatment the patient experienced multiple episodes of déjà vu. Despite déjà vu commonly occurring to some degree in the general population, persistent déjà vu can be related to a neurologic or psychiatric disorder. Déjà vu is a phenomenon which is commonly observed in temporal lobe epilepsy, thus indicating the mesial temporal structures, such as hippocampus, may be related to 'paramnesic' symptoms. Interestingly, Sno et al. [41] have indicated that déjà vu is on a continuum of psychopathology with $\mathrm{RP}$ at the more severe end. Thus, it is plausible to suggest that the continuum of increasing psychopathology from déjà vu to RP is related to increasing DA levels.

Furthermore, an immunomodulatory therapy has been shown to be effective in resolving RP for place in a case study of a 64-year-old male with the rare autoimmune disease, Morvans syndrome [42]. In this case, the patient presented RP following a surgery for bile duct obstruction. Following presentation of RP, he was administered with $0.4 \mathrm{~g} / \mathrm{kg}$ dose of intravenous immunoglobulin (IVIG) for five days, with the addition of $60 \mathrm{mg}$ prednisone after four days. After three days of IVIG treatment, the RP was resolved. Neuropsychology revealed deficits in executive function skills and declarative memory. Neuroimaging data available for this patient showed only white matter age-associated changes. The authors suggest that the resolution of RP following IVIG in this unique case may be related to antibody-mediated voltage-gated potassium channel (VGKC) dysfunction in neurons that are otherwise structurally intact and is pertinent given the favourable response to immunomodulatory therapy which can be implemented in the future for syndromes attributable to VGKC antibody dysfunction.

\section{Conclusions}

Currently our knowledge of RP is limited by our inability to understand irrational thought processes displayed by these patients. The hypotheses and possible mechanisms for the development of RP remain speculative especially considering that similar lesions do not lead 
to the development of RP. However, this only indicates that there may be several mechanisms underlying the psychological, cognitive and neurological aspects of RP with individual differences in these areas resulting in a complex clinical presentation. However, there are some common factors identified so far, including the dominance of right and bifrontal lesions as well as the cognitive disso- nance associated with memory, visuospatial and impaired conceptual integration. With the advent of in vivo neuroimaging, the capacity to investigate which regions are associated with RP is greatly enhanced. Future research should focus on systematic assessment of the development of RP in relation to both its prevalence, conditions under which it develops and extensive in vivo investigations.

\section{References}

1 Pick A: On reduplicative paramnesia. Brain 1903:26:242-267.

- Förstl H, Beats B: Charles Bonnet's description of Cotard's delusion and reduplicative paramnesia in an elderly patient (1788). Br J Psychiatry 1992;160:416-418.

3 Cotard J: Du délire hypocondriaque dans une forme grave de la mélancolie anxieuse. Ann Med Psychol (Paris) 1880;4:168-174.

4 Head H: Aphasia and Kindred Disorders. London: Cambridge University Press. 1926.

5 Paterson A, Zangwill O: Recovery of spatial orientation in the post-traumatic confusional state. Brain 1944;67:54-68.

6 Benson DF, Gardner H, Meadows JC: Reduplicative paramnesia. Neurology. 1976;26: 147-151.

7 Retterstol N: Paranoid psychoses with hypochondriac delusions as the main delusion. A personal follow-up investigation. Acta Psychiatr Scand 1968;44:334-353.

8 Fishbain DA: The frequency of Capgras delusions in a psychiatric emergency service. Psychopathology 1987;20:42-47.

-9 Hakim H, Verma NP, Greiffenstein MF: Pathogenesis of reduplicative paramnesia. J Neurol Neurosurg Psychiatry 1988;51:839841.

10 Pisani A, Marra C, Silveri MC: Anatomical and psychological mechanism of reduplicative misidentification syndromes. Neurol Sci 2000;21:324-328

11 Malloy PF, Richardson ED: The frontal lobes and content-specific delusions. J Neuropsychiatry Clin Neurosci 1994;6:455-466.

-12 Joseph AB, O'Leary DH, Kurland R, et al: Bilateral anterior cortical atrophy and subcortical atrophy in reduplicative paramnesia: a case-control study of computed tomography in 10 patients. Can J Psychiatry 1999;44:685689.

13 Eimer M, Gosling A, Duchaine B: Electrophysiological markers of covert face recognition in developmental prosopagnosia. Brain 2012;135:542-554.

14 Chechlacz M, Rotshtein P, Hansen PC, Riddoch JM, Deb S, Humphreys GW: The neural underpinings of simultanagnosia: disconnecting the visuospatial attention network. J Cogn Neurosci 2012;24:718-735.

15 Box O, Laing H, Kopelman M: The evolution of spontaneous confabulation, delusional misidentification and a related delusion in a case of severe head injury. Neurocase 1999;5: 251-262.

16 Mattioli F, Miozzo A, Vignolo LA: Confabulation and delusional misidentification: a four year follow-up study. Cortex 1999;35: 413-422.

17 DeLuca J, Diamond BJ: Aneurysm of the anterior communicating artery: a review of neuroanatomical and neuropsychological sequelae. J Clin Exp Neuropsychol 1995;17: 100-121.

18 Breier JI, Adair JC, Gold M, Fennell EB, Gilmore RL, Heilman KM: Dissociation of anosognosia for hemiplegia and aphasia during left-hemisphere anesthesia. Neurology 1995;45:65-67.

19 Melzack R: Phantom limbs and the concept of a neuromatrix. Trends Neurosci 1990;13: 88-92.

20 Mikorey M: Phantom and Doppelgänger. Munich, J.F. Lehmanns, 1952.

21 Brugger P, Regard M, Landis T: Illusory reduplication of one's own body: phenomenology and classification of autoscopic phenomena. Cogn Neuropsychiatry 1997;2:19-38.

22 Alexander MP, Stuss DT, Benson DF: Capgras syndrome: a reduplicative phenomenon. Neurology 1979;29:334-339.

23 Cummings JL: Organic delusions. Br J Psychiatry $1985 ; 46: 184-197$.

24 Forstl H, Almeida OP, Owen AM, et al: Psychiatric, neurological and medical aspects of misidentification syndromes: a review of 260 cases. Psychol Med 1991;21:905-910.

25 Benson DF, Djenderedjian A, Miller BL, et al: Neural basis of confabulation. Neurology 1996;46:1239-1243.

26 Feinberg TE, DeLuca J, Giacino JT, et al: Right-hemisphere pathology and the self: delusional misidentification and reduplication; in Feinberg TE, Keenan JP (eds): The Lost Self. New York, Oxford University Press, 2005, pp 100-130.

27 Lee K, Shinbo M, Kanai H, Nagumo Y: Reduplicative paramnesia after a right frontal lesion. Cogn Behav Neurol 2011;24:35-39.

28 Devinsky O: Right cerebral hemisphere dominance for a sense of corporeal and emotional self. Epilepsy Behav 2000;1:60-73.

29 Chabwine JN, Granziera C, Vargas MI, Aboulaffia T, Caratsch L, Schnider A, Landis
T, Perren F: Simultaneous Doppelgänger and limb amputation impression in right frontal opercular stroke. J Neurol Neurosurg Psychiatry 2011;82:1209-1211.

30 Devinsky O: Delusional misidentifications and duplications: right brain lesions, left brain delusions. Neurology 2009 6;72:80-87.

-31 Budson AE, Roth HL, Rentz DM, Ronthal M: Disruption of the ventral visual stream in a case of reduplicative paramnesia. Ann NY Acad Sci 2000;911:447-452.

32 Gainotti G: Face familiarity feelings, the right temporal lobe and the possible underlying neural mechanisms. Brain Res Rev 2007; 56:214-235.

33 Moser DJ, Cohen RA, Malloy PF, Stone WM, Rogg JM: Reduplicative paramnesia: longitudinal neurobehavioral and neuroimaging analysis. J Geriatr Psychiatry Neurol 1998; 11:174-180.

34 Bowles B, Crupi C, Mirsattari SM, et al: Impaired familiarity with preserved recollection after anterior temporal-lobe resection that spares the hippocampus. Proc Natl Acad Sci USA 2007;104:16382-16387.

35 Gainotti G: Emotional behavior and hemispheric side of the lesion. Cortex. 1972;8:4155.

-36 Weinstein EA: The classification of delusional misidentification syndromes. Psychopathology 1994;27:130-135.

37 Ruff RL, Volpe BT: Environmental reduplication associated with right frontal and parietal lobe injury. J Neurol Neurosurg Psychiatry 1981;44:382-386

38 Filley CM, Jarvis PE: Delayed reduplicative paramnesia. Neurology 1987;37:701-703.

- 39 Staton RD, Brumback RA, Wilson H: Reduplicative paramnesia: a disconnection syndrome of memory. Cortex 1982;18:23-35.

40 Taiminen T, Jääskeläinen SK: Intense and recurrent déjà vu experiences related to amantadine and phenylpropanolamine in a healthy male. J Clin Neurosci 2001;8:460462 .

41 Sno HN, Linszen DH, de Jonghe F: Déjà vu experiences and reduplicative paramnesia. Br J Psychiatry 1992;161:565-568.

42 Hudson LA, Rollins YD, Anderson CA, Johnston-Brooks C, Tyler KL, Filley CM: Reduplicative paramnesia in Morvan's syndrome. J Neurol Sci 2008;267:154-157. 